\title{
New application of multimodal ultrasound imaging for identification of myofascial trigger points in the trapezius muscle
}

\author{
Xiao-Ning Liang, Rui-Jun Guo, Shuo Li \\ Department of Ultrasound Medicine, Beijing Chaoyang Hospital, Capital Medical University, Beijing, China \\ Contributions: (I) Conception and design: XN Liang; (II) Administrative support: XN Liang; (III) Provision of study materials or patients: RJ Guo; \\ (IV) Collection and assembly of data: S Li; (V) Data analysis and interpretation: S Li; (VI) Manuscript writing: All authors; (VII) Final approval of \\ manuscript: All authors. \\ Correspondence to: Rui-Jun Guo, MD; Shuo Li, MD. Department of Ultrasound Medicine, Beijing Chaoyang Hospital, Capital Medical University, \\ North Road 8, Gongti, Chaoyang District, Beijing 100020, China. Email: guodaoshi@126.com; lishuo880809@126.com.
}

\begin{abstract}
Background: The study aimed to quantify the characteristics of trapezius myofascial trigger points (MTrPs) using shear wave elastography (SWE) and contrast-enhanced ultrasound (CEUS) and explore the application value of the new ultrasound techniques in identifying MTrPs.

Methods: Forty patients participated in this study. MTrPs in the trapezius muscle were determined by palpation, and SWE and CEUS were used to quantify the focal and adjacent areas. The elastic modulus values and CEUS parameters between the focal area of MTrPs and adjacent areas were evaluated and compared. Pathological biopsy was performed according to the above two methods, and the pathological tissues were observed by Masson staining, immunohistochemistry and electron microscope.
\end{abstract}

Results: The elastic modulus values were significantly higher for the focal area of MTrPs compared to those for adjacent areas $(\mathrm{P}<0.05)$. There were statistically significant differences in $\mathrm{MTrP}$ parameters, including peak intensity, mean transit time, and area between the focal and adjacent areas $(\mathrm{P}<0.05)$. Masson staining showed that there were inflammatory cell infiltration dominated by lymphocytes in the vascular wall. Electron microscopy showed a large number of fibroblast proliferation, lamellar collagen proliferation and lysosomal deposition; immunohistochemical results: the expression of CD3+, CD4+, CD8+, CD68+, mhc$1+$, dys+, CD8 was more than that of CD20 ( $\mathrm{F}=4.385, \mathrm{P}=0.036)$.

Conclusions: Combined use of SWE and CEUS provides a new detection approach for quantitative identification of MTrPs in the trapezius muscle, which has high application value and is a method worthy of wider use in clinical practice.

Keywords: Myofascial trigger points (MTrPs); contrast-enhanced ultrasound (CEUS); shear wave elastography (SWE); elastic modulus

Submitted Jul 15, 2021. Accepted for publication Sep 18, 2021.

doi: 10.21037/apm-21-2103

View this article at: https://dx.doi.org/10.21037/apm-21-2103

\section{Introduction}

Myofascial trigger points (MTrPs) are hyperirritable spots within a taut band of skeletal muscle which can trigger tenderness, characteristic referral pain, motor dysfunction, and autonomic phenomena $(1,2)$. Such diseases are common in people engaged in high-intensity fixed posture labor, such as doctors, teachers, programmers and so on.
In the comprehensive trigger point manual of travel and Simons (3), MTrPs is divided into different types. Active MTrPs produce persistent pain, while latent MTrPs only produce pain during forced palpation (3). At present, the latest research shows that MTrPs, especially the active type, are caused by continuous muscle contraction, promoting hypoxia and ischemia, and then the concentration of substances such as calcitonin gene-related peptide (CGRP) 
and substance $\mathrm{P}$ (SP) increases (4), resulting in pain or dysfunction in a series of peripheral and radiation areas (4-6). At present, the diagnosis of MTrPs primarily relies on manual palpation by clinicians, and the clinical treatment efficacy is mainly evaluated based on subjective evaluation methods, such as visual analog scale (VAS), as there is a lack of effective objective measures. While some studies have shown that MTrPs are in fascia, others have pointed to a muscular origin.

Shear wave elastography (SWE) is a new ultrasoundbased technique that estimates tissue stiffness $(7,8)$, and contrast-enhanced ultrasound (CEUS) has been widely used in the diagnosis of diseases related to the liver, thyroid, breast, and prostate (9-12). In the present study, we used SWE and CEUS to quantify the characteristics of MTrPs in the trapezius muscle and aimed to explore the application value of these new ultrasound techniques in the diagnosis of MTrPs. We present the following article in accordance with the STROBE reporting checklist (available at https:// dx.doi.org/10.21037/apm-21-2103).

\section{Methods}

\section{Subjects}

Between April and June 2019, 40 patients presenting with back pain (excluding fibromyalgia and joint dysfunction), whose age ranged from 40 to 76 (mean $47.67 \pm 5.79$ ) years, were included in this study. The palpatory diagnosis of MTrPs was conducted by an experienced physician based using the Simons diagnostic criteria for MTrPs (2), and a total of $30 \mathrm{MTrPs}$ in the trapezius muscle were detected (excluding in the rhomboid and serratus anterior muscles). A statement that the participants gave informed consent before taking part and ethical approval was obtained (2019ke-19). The study was conducted in accordance with the Declaration of Helsinki (as revised in 2013).

\section{$S W E$}

SWE was performed by an experienced radiologist using an Aplio 500 color Doppler diagnostic ultrasound system (Toshiba Medical Systems Corporation, Tochigi, Japan), with a high-frequency $(5-14 \mathrm{MHz})$ probe, and an elastic modulus value ranging from 0 to $100 \mathrm{kPa}$. Patients were placed in the supine position with their hands naturally placed on both sides of the body to allow the muscles to be as relaxed as possible. Based on observations in the gray scale ultrasound, the scanner was switched to the SWE mode and the elasticity imaging area was set. The probe was kept motionless for a few seconds to stabilize the SWE images, which were then frozen. The focal area of the MTrPs was determined based on the area with higher elastic modulus, then the adjacent area (along the muscle fiber direction) within the same muscle was chosen as regionof-interest (ROI). In addition, the same positions on the healthy side were selected. A quantitative analysis sampling frame Q-box was placed in the focal and the adjacent areas, with Q-box diameters set as $2 \mathrm{~mm}$, while the maximum, minimum, and mean of the elastic modulus in the sampling frame were calculated automatically. Each MTrP site was measured three times and the mean value was taken for analysis.

\section{CEUS}

CEUS was performed using an Aplio 500 color Doppler diagnostic ultrasound system (Toshiba Medical Systems Corporation, Tochigi, Japan) with a high-frequency (5-14 MHz) probe, and the mechanical index (MI) set at 0.08. The contrast agent used was SonoVue (Bracco SpA, Milan, Italy) which, prior to use, was diluted with $5 \mathrm{~mL}$ of physiological saline and repeatedly agitated to form a white SF6 microbubble suspension. After multi-section conventional ultrasound of the trapezius muscle was performed, the mode was switched to contrast ultrasound imaging mode. For this, $2.4 \mathrm{~mL}$ contrast agent was injected as a rapid bolus, followed by $5 \mathrm{~mL}$ of saline while maintaining the same observation view. Simultaneously with injection, the timer was started, the dynamic process of lesion perfusion was observed continuously in real-time with observation time $\geq 120 \mathrm{~s}$, and the image then saved. Dynamic images were replayed, and the data was recorded.

\section{Biopsy for pathology}

Take pathological biopsy according to the ultrasonic elasticity and CEUS area, use Toshiba Apollo 500 color Doppler ultrasound diagnostic instrument, high-frequency probe, frequency $5-14 \mathrm{MHz}$, explain the condition and surgical complications to the family members and patients, and sign the informed consent. Select the best needle entry path and mark it on the body surface. Routine disinfection, towel laying, reconfirming the puncture point, and local infiltration anesthesia with $10 \mathrm{~mL}$ of $2 \%$ lidocaine at the puncture point. The needle was quickly inserted into 

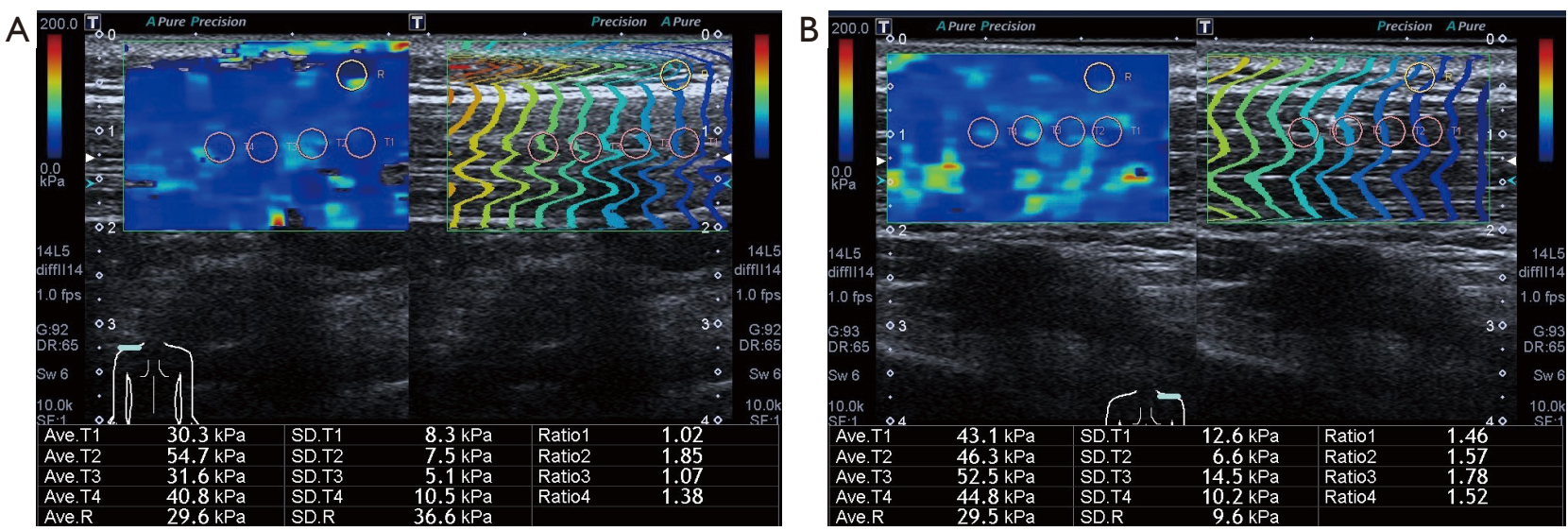

Figure 1 Measurement of elastic modulus of trapezius muscle. (A) Measurement of elastic modulus of trapezius muscle in the focal (T2), adjacent (T1, T3, T4), and superficial fascia (R) areas on the affected side. (B) Measurement of elastic modulus of trapezius muscle in the healthy area (T2), area adjacent to the healthy area (T1, T3, T4), and superficial fascia (R) areas on the healthy side.

the area jointly determined by ultrasound elasticity and CEUS. Ultrasound was used to observe whether there was active bleeding and local dressing coverage. Ask the patient to avoid strenuous activities and return to the ward. The pathological tissues were observed by Masson staining, immunohistochemistry and electron microscope.

\section{Statistical analysis}

Statistical analyses were performed using SPSS version 22.0. Measurement data are expressed as mean \pm standard deviation (mean $\pm \mathrm{SD}$ ). Paired $t$-test was used to compare the difference in elastic modulus within groups, and oneway ANOVA was used to compare the differences in elastic modulus between groups. A P level of $<0.05$ was considered statistically significant.

\section{Results}

\section{Elastic modulus of the trapezius muscle}

The mean of the elastic modulus in the focal area of MTrPs was significantly higher compared to that in the adjacent area $(\mathrm{t}=4.352, \mathrm{P}<0.001)$, as well as in the superficial fascia $(\mathrm{t}=6.878, \mathrm{P}<0.001)$. The mean of the elastic modulus in adjacent muscular layers was also significantly higher than those in the superficial fascia $(\mathrm{t}=5.771, \mathrm{P}<0.001)$ (Figure 1). As no MTrPs were detected on the healthy side, there were no statistically significant differences in the mean of the elastic modulus of the trapezius muscle between the healthy area and adjacent healthy area $(\mathrm{t}=1.171, \mathrm{P}=0.251)$. However, statistically significant differences were found between the healthy area, adjacent healthy area and superficial fascia, respectively, on the healthy side $(\mathrm{t}=8.271, \mathrm{P}<0.001 ; \mathrm{t}=6.985$, $\mathrm{P}<0.001$ ) (Figure 2). Comparison of the elastic modulus in areas of MTrPs and the same position on the healthy side are shown in Table 1. While there were no statistically significant differences in the elastic modulus in the fascial layer between affected and healthy sides, there were significant differences in the elastic modulus between the focal, adjacent areas of MTrPs and the same areas on the healthy side, respectively (both $\mathrm{P}<0.05$ ).

\section{CEUS results in focal area of MTrP and its adjacent area}

Results from CEUS in the focal and adjacent areas of MTrPs are shown in Table 2. The peak intensity, mean transit time, and area under the curve of the focal area were higher than those of the adjacent area (Figure 3), and there were no significant differences in time to peak and slope between the focal area of MTrPs and their adjacent area.

\section{Pathological results}

According to the ultrasound elasticity and CEUS localization, the pathology was taken for Masson staining, electron microscopic observation and immunohistochemistry (Figure 4A-4H).

Masson staining showed that the striated muscle showed mild atrophy ( 3 cases, 10\%), moderate atrophy (17 cases, $56.7 \%)$, severe atrophy (10 cases, $33.3 \%)$, and lymphocyte 


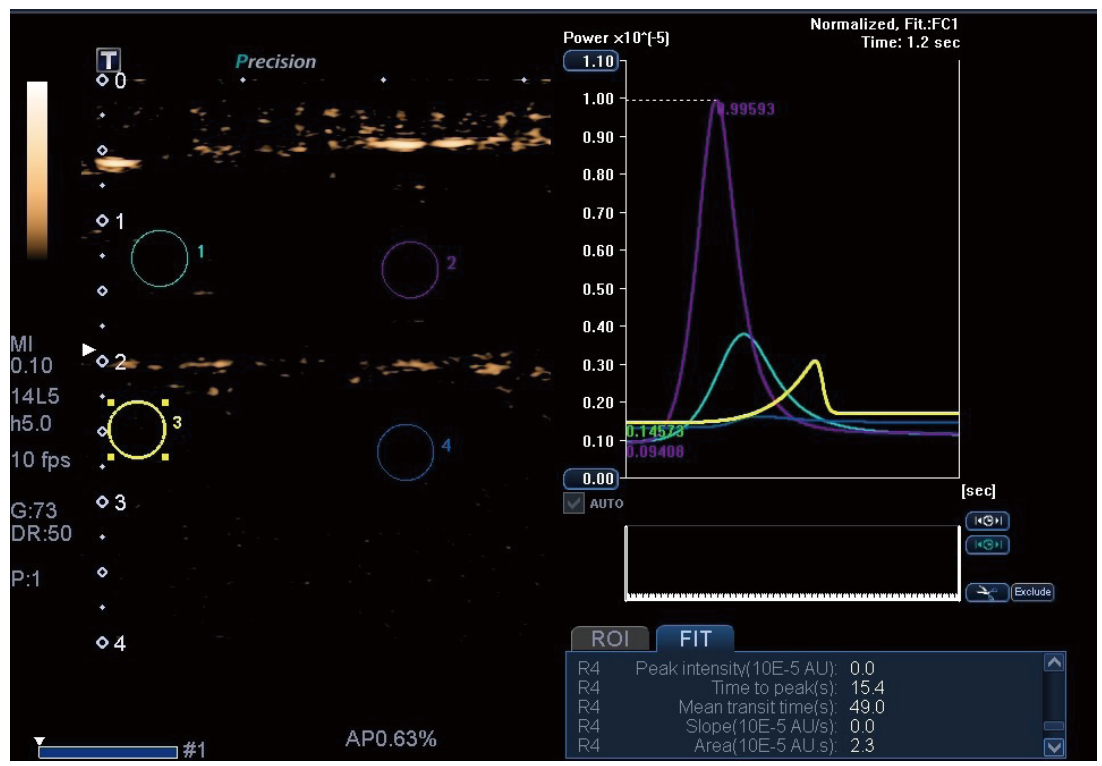

Figure 2 Receiver operating characteristic curve obtained from CEUS images. Number 2 (pink) indicates the focal area of the trapezius MTrPs, and number 1 (green) indicates the adjacent area. CEUS, contrast-enhanced ultrasound; MTrPs, myofascial trigger points.

Table 1 Comparison of the elastic modulus in areas of the trapezoid muscle MTrPs and the control side (mean $\pm \mathrm{SD}, \mathrm{kPa})$

\begin{tabular}{|c|c|c|c|c|}
\hline Different locations of trapezius muscle & Affected side & Healthy side & t value & $P$ value \\
\hline Adjacent area & $35.95 \pm 21.97$ & $36.04 \pm 17.67$ & 3.204 & 0.003 \\
\hline Fascial layer & $22.67 \pm 12.77$ & $26.83 \pm 12.91$ & 0.298 & 0.768 \\
\hline$F$ value & 30.434 & 35.125 & & \\
\hline
\end{tabular}

MTrPs, myofascial trigger points; SD, standard deviation.

Table 2 Comparison of CEUS results in focal and adjacent areas of the trapezoid muscle MTrPs (mean \pm SD)

\begin{tabular}{lccccc}
\hline locations & Peak intensity & Time to peak $(\mathrm{s})$ & Mean transit time $(\mathrm{s})$ & Slope & Area under the curve $\left(\mathrm{cm}^{2}\right)$ \\
\hline Focal area & $7.42 \pm 5.10$ & $15.19 \pm 5.99$ & $24.17 \pm 16.41$ & $1.12 \pm 1.18$ & $42.70 \pm 18.36$ \\
Adjacent area & $2.30 \pm 2.22$ & $13.04 \pm 6.21$ & $11.58 \pm 4.53$ & $1.61 \pm 1.00$ & $25.61 \pm 11.56$ \\
$t$ value & 5.98 & 1.465 & 4.307 & 1.550 & 4.550 \\
$P$ value & $<0.001$ & 0.154 & $<0.001$ & 0.132 & $<0.001$ \\
\hline
\end{tabular}

CEUS, contrast-enhanced ultrasound; MTrPs, myofascial trigger points; SD, standard deviation.

dominated inflammatory cell infiltration in the vascular wall (30 cases, $100 \%)$.

Electron microscopic results: simple atrophy of striated muscle (6 cases, $20 \%$ ); fibroblast proliferation (24 cases, $80 \%)$; lamellar collagen hyperplasia (24 cases, $80 \%$ ); fibroblast endoplasmic reticulum dilation and edema (6 cases, 20\%); lysosomal deposition (30 cases, 100\%); mitochondria increased (6 cases, 20\%).

Immunohistochemical results: CD3 (in vascular wall and stroma, positive in 30 cases, $100 \%$ ); CD4 (vascular 


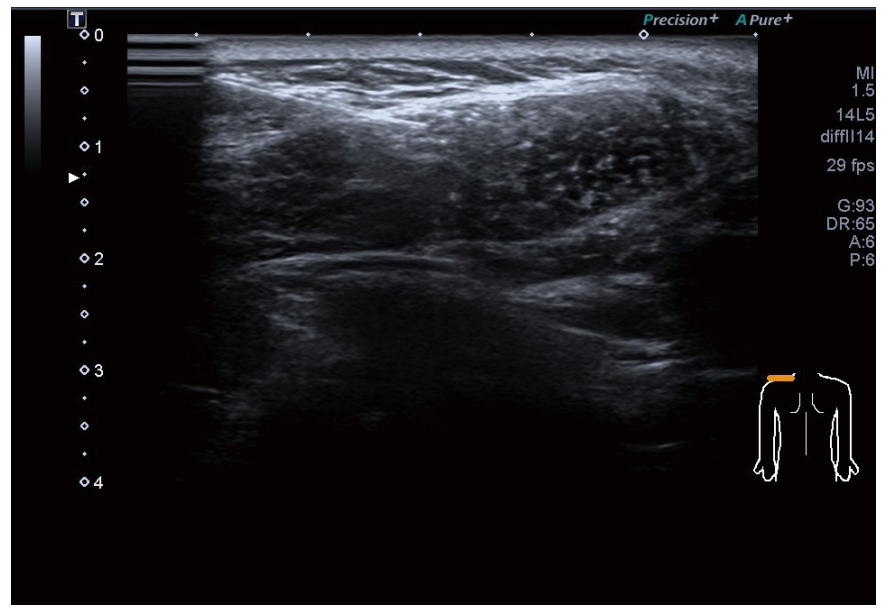

Figure 3 Ultrasound guided biopsy of the left trapezius muscle pain point.
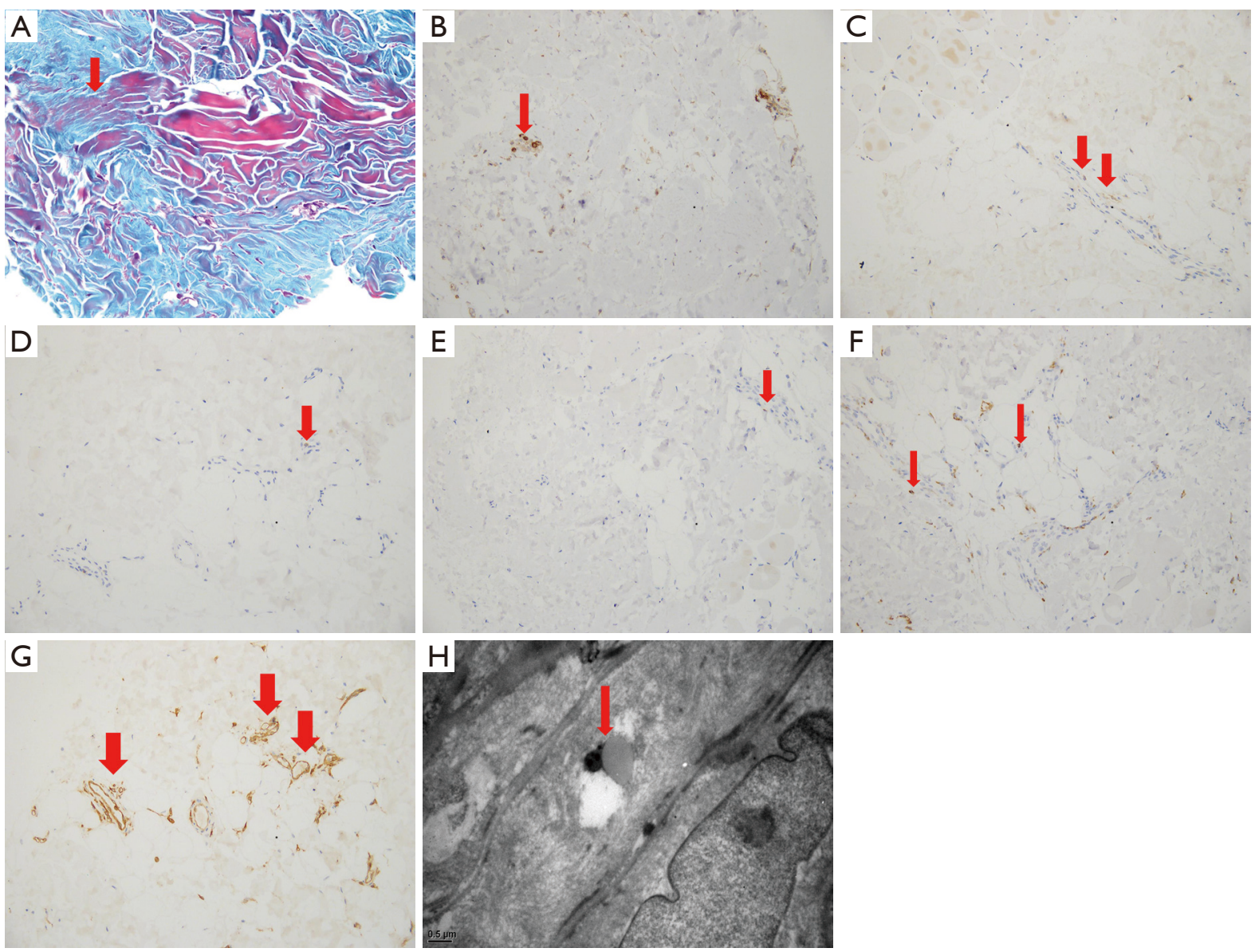

Figure 4 Pathological images were obtained according to ultrasound elasticity and CEUS localization. Masson staining, electron microscopy, and immunohistochemistry were performed. (A) Masson staining (zoom in 100 times), red arrow: muscle atrophy; (B-G) CD3 (zoom in 200 times), CD4 (zoom in 100 times), CD8 (zoom in 100 times), CD20 (zoom in 100 times), CD68 (zoom in 100 times), and HLA-DR immunohistochemistry (zoom in 100 times); red arrows: immunohistochemical positive; (H) electron micrograph, red arrow: lysosomal deposition. CEUS, contrast-enhanced ultrasound. 
Table 3 Comparison of CD8 and CD20

\begin{tabular}{lccc}
\hline \multirow{2}{*}{ CD8 } & \multicolumn{3}{c}{ CD20 } \\
\cline { 2 - 4 } & - & & + \\
\hline- & 1 & & 4 \\
+ & 17 & 7 \\
$\mathrm{~F}$ & & 4.385 & \\
$\mathrm{P}$ & & 0.036 & \\
\hline
\end{tabular}

wall, positive in 30 cases, 100\%); CD8 (perivascular, positive in 24 cases, $80 \%$ ); HLA-DR (vascular endothelial and inflammatory cells, positive in 16 cases, $53.3 \%$ ); CD68 (perivascular, positive in 30 cases, 100\%); CD20 (perivascular, positive in 12 cases, 40\%); Mhc-1 (NS 30 cases, 100\%), dys (NS 30 cases, 100\%). See Table 3 for the comparison between CD8 and CD20. The expression of CD8 is more than that of CD20. It suggests that T cells are involved in the process of disease.

\section{Discussion}

At present, the diagnosis of MTrPs is mainly based on the diagnostic criteria described by Simons et al. $(1,2)$, which includes the presence of a palpable taut band in a skeletal muscle, tenderness in a hypersensitive spot within a palpable taut band, complaints of pain, and limited range of motion. However, MTrPs are mainly identified by palpation, which may be easily influenced by subjective factors, has low interexaminer reliability, and is difficult to conduct when MTrPs are deep, or the lesion is small. This study confirmed that ultrasound method is a significant improvement, which can use relatively cheap equipment to image myofascial pain points in ordinary consulting rooms. In addition to the traditional two-dimensional gray-scale imaging, the use of ultrasound elasticity and CEUS provides a quantitative standard for the evaluation of MTrPs. In addition, ultrasound elastography may be more sensitive than 2D imaging in distinguishing MTrPs from surrounding muscles. In the present study, the results showed that the mean of the elastic modulus in the focal area of MTrPs was significantly higher compared to the adjacent area $(\mathrm{t}=4.352$, $\mathrm{P}<0.001)$, as well as in the superficial fascia $(\mathrm{t}=6.878$, $\mathrm{P}<0.001)$. The mean of the elastic modulus in adjacent muscular layers was also significantly higher than that in the superficial fascia $(t=5.771, \mathrm{P}<0.001)$. As there were no MTrPs on the control side, there were no statistically significant differences in the mean of the elastic modulus between the healthy area and adjacent healthy area within the same muscle on the healthy side $(\mathrm{t}=1.171, \mathrm{P}=0.251)$. However, there were statistically significant differences between the healthy, adjacent control areas and superficial fascia, respectively, on the healthy side $(\mathrm{t}=8.271, \mathrm{P}<0.001$; $\mathrm{t}=6.985, \mathrm{P}<0.001)$. This suggests the elastic modulus in the focal area of MTrPs was significantly increased compared to those in the adjacent area, which means the hardness of the focal area was significantly higher than that of the adjacent area. In addition, the CEUS showed that the peak intensity, mean transit time, and area under the curve of the focal area were higher than those of the adjacent area. The results confirmed that MTrPs are present inside the muscle, not the superficial fascia. Although our findings provide a scientific basis for MTrPs palpation, how to apply this to patient diagnosis in a standardized way requires further research.

SWE can display color-coded images of tissue elasticity with the guidance of two-dimensional gray-scale ultrasound, and the system automatically calculates the elastic modulus values of the corresponding area. SWE is considered a simple and convenient technique and has moderate cost, making it suitable for the diagnosis of MTrPs in a pain clinic. SWE can directly obtain the elastic modulus of the tissue and reflect the tissue hardness. It also has the advantage of not requiring pressure by the operator, realtime two-dimensional imaging guidance, quantitative detection, measurement results that are not influenced by the operator, and good repeatability (13-16). SWE has been widely used in the diagnosis of lesions in the liver, thyroid, breast, prostate, kidney, and blood vessels (17-19). Studies have investigated the morphological changes and microcirculation of MTrPs using gray-scale ultrasound and color Doppler technology (20-23), but the use of ultrasound and SWE for MTrP identification has not been reported in China.

In this study, the CEUS results showed there were statistically significant differences in the peak intensity, average transit time, and area between the focal and adjacent areas of MTrPs, indicating that the blood supply to there is greater than that to the adjacent area, and there were enhancement differences between the two. Timeintensity curves obtained with CEUS can provide indirect information about blood flow inside the lesion as those with different vascular structures and different microcirculation perfusion states show different contrast enhancement patterns. 
In conclusion, SWE could detect the elastic modulus of trigger points in the trapezius muscle, and CEUS could determine painful areas and display blood perfusion at lesion sites. SWE combined with CEUS provides a new detection approach for quantitative identification of $\mathrm{MTrPs}$ in the trapezius muscle and has high reliability. In addition, this new ultrasound technology can be further applied to guide the depth of needle insertion in acupuncture practice, and objectively evaluate the efficacy of dry needling, laser acupuncture, and distraction in the treatment of MTrPs, which has high application value and broad prospects for future development.

\section{Acknowledgments}

Funding: None.

\section{Footnote}

Reporting Checklist: The authors have completed the STROBE reporting checklist. Available at https://dx.doi. org/10.21037/apm-21-2103

Data Sharing Statement: Available at https://dx.doi. org/10.21037/apm-21-2103

Conflicts of Interest: All authors have completed the ICMJE uniform disclosure form (available at https://dx.doi. org/10.21037/apm-21-2103). The authors have no conflicts of interest to declare.

Ethical Statement: The authors are accountable for all aspects of the work in ensuring that questions related to the accuracy or integrity of any part of the work are appropriately investigated and resolved. A statement that the participants gave informed consent before taking part and ethical approval was obtained (2019-ke-19). The study was conducted in accordance with the Declaration of Helsinki (as revised in 2013).

Open Access Statement: This is an Open Access article distributed in accordance with the Creative Commons Attribution-NonCommercial-NoDerivs 4.0 International License (CC BY-NC-ND 4.0), which permits the noncommercial replication and distribution of the article with the strict proviso that no changes or edits are made and the original work is properly cited (including links to both the formal publication through the relevant DOI and the license).
See: https://creativecommons.org/licenses/by-nc-nd/4.0/.

\section{References}

1. Mazza DF, Boutin RD, Chaudhari AJ. Assessment of myofascial trigger points via imaging: a systematic review. Am J Phys Med Rehabil 2021;100:1003-14.

2. Shah JP, Thaker N, Heimur J, et al. Myofascial trigger points then and now: a historical and scientific perspective. PM R 2015;7:746-61.

3. Simons D, Travell J. Travell \& Simons' myofascial pain and dysfunction: the trigger point manual. Baltimore: Williams \& Wilkins, 1999.

4. Travell J, Rinzler SH. The myofascial genesis of pain. Postgrad Med 1952;11:425-34.

5. Stockman R. The causes, pathology, and treatment of chronic rheumatism. Edinb Med J 1904;15:223.

6. Fernández-de-las-Peñas C, Dommerholt J. Myofascial trigger points: peripheral or central phenomenon? Curr Rheumatol Rep 2014;16:395.

7. Shah JP, Danoff JV, Desai MJ, et al. Biochemicals associated with pain and inflammation are elevated in sites near to and remote from active myofascial trigger points. Arch Phys Med Rehabil 2008;89:16-23.

8. Shah JP, Phillips TM, Danoff JV, et al. An in vivo microanalytical technique for measuring the local biochemical milieu of human skeletal muscle. J Appl Physiol (1985) 2005;99:1977-84.

9. Sikdar S, Shah JP, Gebreab T, et al. Novel applications of ultrasound technology to visualize and characterize myofascial trigger points and surrounding soft tissue. Arch Phys Med Rehabil 2009;90:1829-38.

10. Malanga GA, Cruz Colon EJ. Myofascial low back pain: a review. Phys Med Rehabil Clin N Am 2010;21:711-24.

11. Clauser P, Baltzer PAT, Kapetas P, et al. Low-dose, contrast-enhanced mammography compared to contrastenhanced breast MRI: a feasibility study. J Magn Reson Imaging 2020;52:589-95.

12. Wilson SR, Burns PN, Kono Y. Contrast-enhanced ultrasound of focal liver masses: a success story. Ultrasound Med Biol 2020;46:1059-70.

13. Rana SS, Sharma R, Srinivasan R, et al. Contrast-enhanced EUS in the evaluation of peritoneum and omentum in undiagnosed ascites. Endosc Ultrasound 2020;9:69-70.

14. Qiu X, Zhao Q, Ye Z, et al. How does contrast-enhanced ultrasonography influence Bosniak classification for complex cystic renal mass compared with conventional ultrasonography? Medicine (Baltimore) 2020;99:e19190. 
15. Terashi E, Kodama Y, Kuraoka A, et al. Usefulness of liver stiffness on ultrasound shear-wave elastography for the evaluation of central venous pressure in children with heart diseases. Circ J 2019;83:1338-41.

16. Zhu J, He X, Chen Z. Acoustic radiation force optical coherence elastography for elasticity assessment of soft tissues. Appl Spectrosc Rev 2019;54:457-81.

17. Dong L, Wijesinghe P, Sampson DD, et al. Volumetric quantitative optical coherence elastography with an iterative inversion method. Biomed Opt Express 2019;10:384-98.

18. Zhu J, Yu J, Qu Y, et al. Coaxial excitation longitudinal shear wave measurement for quantitative elasticity assessment using phase-resolved optical coherence elastography. Opt Lett 2018;43:2388-91.

19. Liu B, Liang J, Zheng Y, et al. Two-dimensional shear wave elastography as promising diagnostic tool for predicting malignant thyroid nodules: a prospective singlecentre experience. Eur Radiol 2015;25:624-34.

20. Elkrief L, Rautou PE, Ronot M, et al. Prospective comparison of spleen and liver stiffness by using shear-

Cite this article as: Liang XN, Guo RJ, Li S. New application of multimodal ultrasound imaging for identification of myofascial trigger points in the trapezius muscle. Ann Palliat Med 2021;10(9):9784-9791. doi: 10.21037/apm-21-2103 wave and transient elastography for detection of portal hypertension in cirrhosis. Radiology 2015;275:589-98.

21. Koszalinski A, Flynn T, Hellman M, et al. Trigger point dry needling, manual therapy and exercise versus manual therapy and exercise for the management of Achilles tendinopathy: a feasibility study. J Man Manip Ther 2020;28:212-21.

22. Liu S, Wang L, Yang J. Instant analgesic effect of radial extracorporeal shock wave therapy on primary dysmenorrhoea according to functional magnetic resonance imaging: study protocol for a randomised placebo-controlled trial. Trials 2020;21:164.

23. Kodama K, Takamoto K, Nishimaru H, et al. Corrigendum: analgesic effects of compression at trigger points are associated with reduction of frontal polar cortical activity as well as functional connectivity between the frontal polar area and insula in patients with chronic low back pain: a randomized trial. Front Syst Neurosci 2020;13:81. Erratum for: Front Syst Neurosci 2019;13:68.

(English Language Editor: B. Draper) 